\title{
Erratum to: Structural and functional analyses of human DDX41 DEAD domain
}

\author{
Yan Jiang ${ }^{1}$, Yanping Zhu' ${ }^{1}$, Weicheng Qiu ${ }^{1}$, Yong-Jun Liu ${ }^{2}$, Genhong Cheng ${ }^{3}$, Zhi-Jie Liu ${ }^{1,4 \bowtie}$, \\ Songying Ouyang ${ }^{1,3 \bowtie}$
}

${ }^{1}$ National Laboratory of Biomacromolecules, Institute of Biophysics, Chinese Academy of Sciences, Beijing 100101, China

2 Baylor Research Institute, Baylor Scott and White Health, Dallas, TX 75246, USA

${ }^{3}$ Department of Microbiology, Immunology and Molecular Genetics, University of California Los Angeles, Los Angeles, CA 90095, USA

${ }^{4}$ Institute of Molecular and Clinical Medicine, Kunming Medical University, Kunming 650500, China

$\bowtie$ Correspondence: zjliu@ibp.ac.cn (Z.-J. Liu), ouyangsy@ibp.ac.cn (Songying Ouyang)

\section{ERRATUM TO: PROTEIN CELL 2016 \\ DOI 10.1007/S13238-016-0351-9}

In the original publication of this article Fig. 2 has been incorrectly published. The correct Fig. 2 is provided in this erratum. 


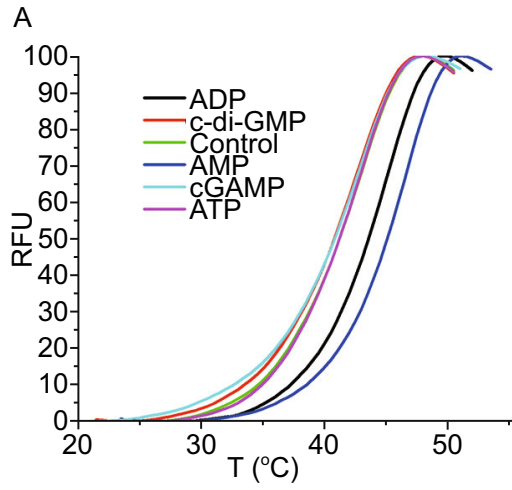

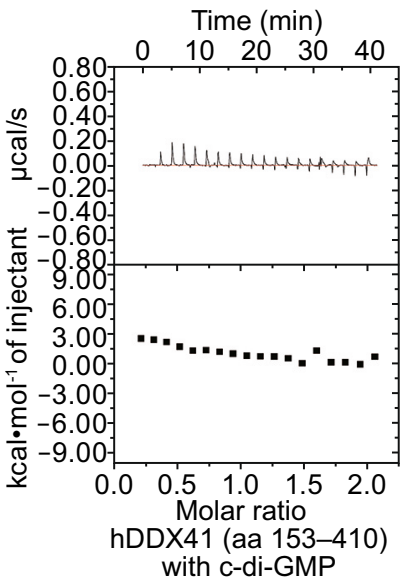
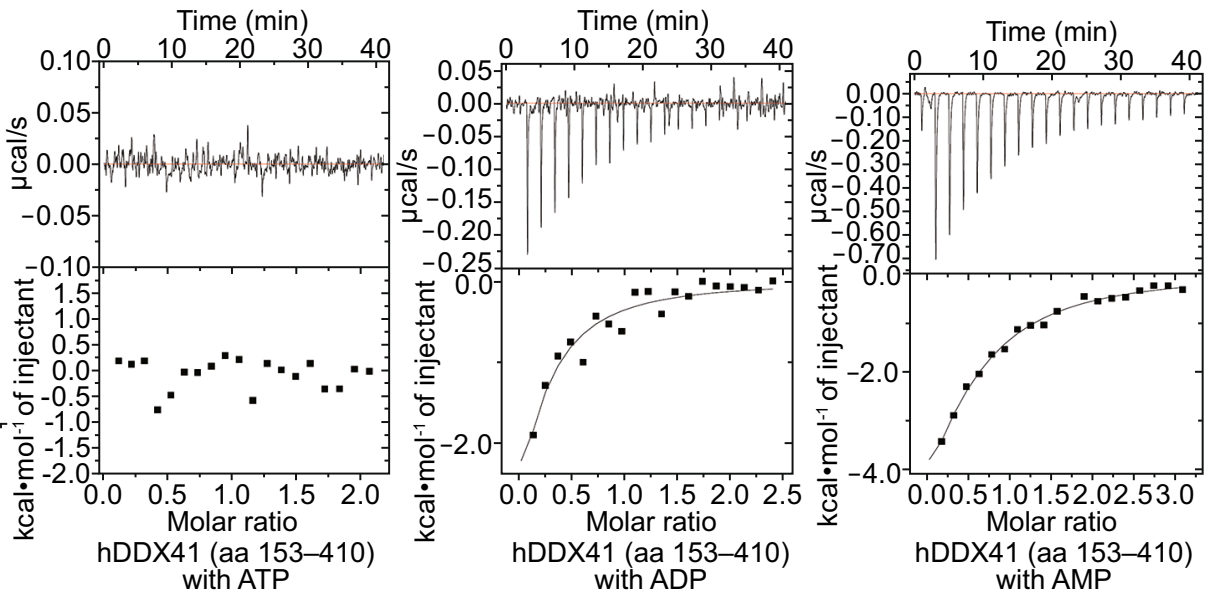

C

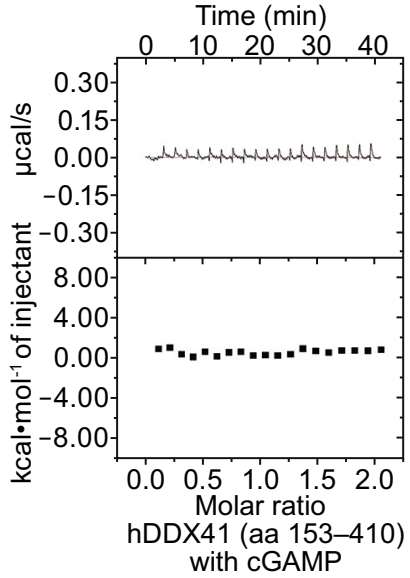

B with ADP with AMP
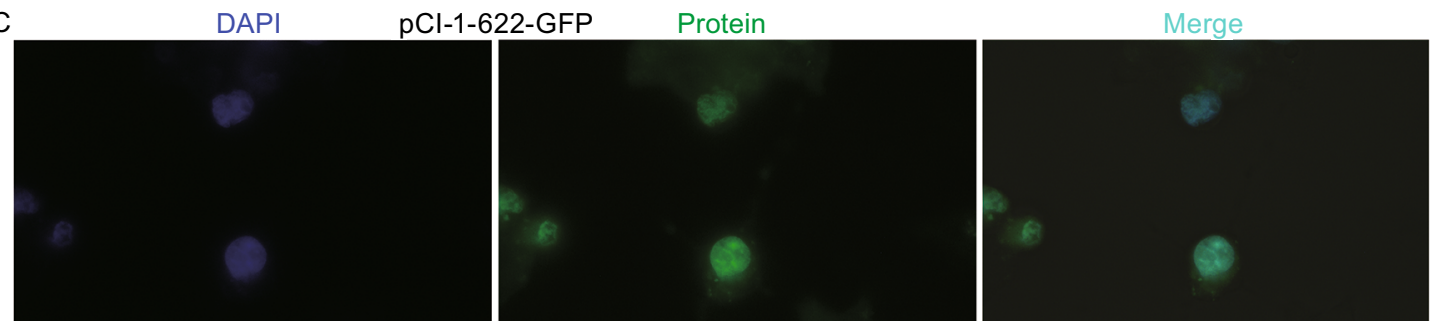

DAPI

pCl-153-622-GFP

Protein

Merge


Figure 2. The binding of hDDX41 DEAD domain with different molecules and N-terminal region targets hDDX41 to the nucleus. (A) Thermal Shift Assay and Isothermal Titration Calorimetry of hDDX41 DEAD domain protein with ATP, ADP, AMP, c-diGMP and cGAMP. (B) Left: the modeled ADP and ANP are colored in cyan and green. The y-phosphate of ANP clashes with T227 of hDDX41. Right: surface electrostatic potential representation of the nucleotide binding pocket. Blue, positive potential; red, negative potential. The positively charged binding pocket is not big enough for ANP binding. (C) Fluorescence microscopy of HEK293T cells transfected with expression plasmids for GFP-tagged hDDX41 full length protein (1-622) and GFP-tagged hDDX41 N-terminal region deleted truncation (153-622). Nuclei are stained with DAPI. 


\section{OPEN ACCESS}

This article is distributed under the terms of the Creative Commons Attribution 4.0 International License (http://creativecommons.org/ licenses/by/4.0/), which permits unrestricted use, distribution, and reproduction in any medium, provided you give appropriate credit to the original author(s) and the source, provide a link to the Creative Commons license, and indicate if changes were made. 